\title{
Kink plateau dynamics in finite-size lubricant chains
}

\author{
Marco Cesaratto ${ }^{a}$, Nicola Manini $^{a}$, Andrea Vanossi ${ }^{b}$, \\ Erio Tosatti ${ }^{c, d}$, and Giuseppe E. Santoro ${ }^{c, d *}$ \\ ${ }^{a}$ Department of Physics, University of Milan, Via Celoria 16, 20133 Milan, Italy \\ ${ }^{b}$ CNR-INFM National Research Center S3, and Department of Physics, University \\ of Modena and Reggio Emilia, Via Campi 213/A, 41100 Modena, Italy \\ ${ }^{c}$ International School for Advanced Studies (SISSA), and INFM-CNR Democritos \\ National Simulation Center, Via Beirut 2-4, I-34014 Trieste, Italy \\ ${ }^{d}$ International Centre for Theoretical Physics (ICTP), P.O.Box 586, I-34014 \\ Trieste, Italy
}

\begin{abstract}
We extend the study of velocity quantization phenomena recently found in the classical motion of an idealized 1D model solid lubricant - consisting of a harmonic chain interposed between two periodic sliding potentials [Phys. Rev. Lett. 97, 056101 (2006)]. This quantization is due to one slider rigidly dragging the commensurate lattice of kinks that the chain forms with the other slider. In this follow-up work we consider finite-size chains rather than infinite chains. The finite size (i) permits the development of robust velocity plateaus as a function of the lubricant stiffness, and (ii) allows an overall chain-length re-adjustment which spontaneously promotes single-particle periodic oscillations. These periodic oscillations replace the quasiperiodic motion produced by general incommensurate periods of the sliders and the lubricant in the infinite-size model. Possible consequences of these results for some real systems are discussed.
\end{abstract}

\section{Introduction}

The present paper extends the study of a one-dimensional (1D) non-linear model, inspired by the tribological problem of two sliding surfaces with a thin

* Corresponding Author.

Email address: santoro@sissa.it (Giuseppe E. Santoro ${ }^{c, d}$ ). 
solid lubricant layer in between [1], to the case of a lubricant 1D "island" of finite size. Previous work $[1,2]$ found robust, universal and exactly quantized asymmetric velocity plateaus in the classical dynamics of an infinite-size chain subject to two relatively sliding periodic potentials. The infinite chain size was managed - in the general incommensurate case - by means of periodic boundary conditions (PBC) and finite-size scaling. The plateaus of chain velocity as a function of several model parameters were shown to be due to motion of kinks (topological solitons), which generally exist in a chain submitted to a periodic potential. These nonlinear excitations generated by the first, stationary periodic potential are set into motion by the external driving which is provided by the second periodic potential, sliding with velocity $v_{\text {ext }}$. While the chain kinks are thus dragged with velocity $v_{\text {ext }}$, the overall chain velocity is smaller, and fixed by the kinks nature and density. That in turn depends only on the ratio of the period of one slider to that of the chain, which is dictated by the interparticle spacing, and enforced by the PBC.

The present work considers a finite open-boundary chain, such as for example a hydrocarbon chain, or a graphite flake interposed between two sliding crystal faces [3]. Unlike the infinite chain, the open-boundary chain can elongate or shorten, at the cost of some harmonic potential energy, effectively modifying its linear density, and thus the kink density which is the relevant length ratio. We find that it does indeed elongate or shorten so as to realize a precise commensurate relation to the other slider. This adaptive relaxation is such as to produce perfectly periodic oscillations of the single particles superposed with their average drift at the quantized velocity.

\section{The model}

Using the same language and notation of previously studied confined models $[4,5,6,7,8]$, we represent a solid lubricant layer as a chain of $N$ harmonically interacting particles interposed between two rigid generally (but not necessarily) incommensurate sinusoidal substrates (the two "sliding crystals", sketched in Fig. 1) externally driven at a constant relative velocity $v_{\text {ext }}$. The equation of motion of the $i$-th particle is:

$$
\begin{aligned}
m \ddot{x}_{i} & =-\frac{1}{2}\left[F_{+} \sin k_{+}\left(x_{i}-v_{+} t\right)+F_{-} \sin k_{-}\left(x_{i}-v_{-} t\right)\right] \\
& +K\left(x_{i+1}+x_{i-1}-2 x_{i}\right)-\gamma \sum_{ \pm}\left(\dot{x}_{i}-v_{ \pm}\right)
\end{aligned}
$$

where $m$ is the mass of the $N$ particles, $K$ is the chain spring constant, and $k_{ \pm}=2 \pi / a_{ \pm}$are the wave-vector periodicities of potentials representing the two sliders, moving at velocities $v_{ \pm}$. We set, in full generality, $v_{+}=0$ and 
$v_{-}=v_{\text {ext }} \cdot \gamma$ is a phenomenological parameter substituting for various sources of dissipation, required to achieve a stationary state, but otherwise playing no major role in the following. $F_{ \pm}$are the force amplitudes representing the sinusoidal corrugation of the two sliders (we will commonly use $F_{-} / F_{+}=1$ but we checked that our results are more general). We take $a_{+}=1, m=1$, and $F_{+}=1$ as our basic units, and all quantities are measured in suitable combinations thereof. The relevant length ratios $[9,10]$ are defined by $r_{ \pm}=$ $a_{ \pm} / a_{0}$; we assume, without loss of generality, $r_{-}>r_{+}$. The inter-particle equilibrium length $a_{0}$ enters explicitly the equations of motion (1) of the first $(i=1)$ and last $(i=N)$ particle whose restoring force terms in Eq. (1) are $K\left(x_{2}-x_{1}-a_{0}\right)$ and $K\left(x_{N-1}-x_{N}+a_{0}\right)$, respectively; this implements open boundary conditions (OBC).

Upon sliding the substrates, $v_{\text {ext }} \neq 0$, the lubricant chain slides too. Despite the apparent generic symmetry between the two sliders, the time-averaged chain velocity $w=v_{\mathrm{cm}} / v_{\text {ext }}$, is generally asymmetric, namely different from $1 / 2$. In a previous study on this model $[1,2]$ it was shown that, for an infinite chain and periodic boundary conditions (PBC), $w$ is exactly quantized, for large parameter intervals, to plateau values that depend solely on the chosen commensurability ratios $\left(r_{+}, r_{-}\right)$. As the present finite-size OBC simulations will show, the PBCs are not crucial to the plateau quantization, which occurs even for a lubricant of finite and not particularly large size $N$. The main difference between $\mathrm{OBC}$ and $\mathrm{PBC}$ is that, while in $\mathrm{PBC}$ the chain length, and thus the length ratios $r_{ \pm}$, are fixed, in $\mathrm{OBC}$ the chain can lengthen or shorten with respect to its equilibrium size. We find that during sliding the chain length gradually reaches a natural attractor value and oscillates around it. We define a new effective inter-particle length and corresponding length ratio

$$
a_{0}^{\mathrm{eff}}=\frac{L}{N-1}, \quad r_{+}^{\mathrm{eff}}=\frac{a_{+}}{a_{0}^{\mathrm{eff}}}
$$

where $L=\left\langle x_{N}-x_{1}\right\rangle$ is the average chain length after the initial transient. The effective length ratio $r_{+}^{\text {eff }}$ plays a central role in the understanding of the velocity plateaus of the finite-size lubrication model.

\section{Results and theory}

The driven dynamics of the lubricant is studied by integrating the equations of motion (1) starting from fully relaxed springs $\left(x_{i}=i a_{0}, \dot{x}_{i}=v_{\text {ext }} / 2\right)$, using a standard fourth-order Runge-Kutta method. After an initial transient where length relaxation takes place, the system reaches its dynamical stationary state, at least so long as $\gamma$ is not exactly zero. Figure 2 shows the resulting 
time-averaged center-of-mass $(\mathrm{CM})$ velocity $v_{\mathrm{cm}}$ as a function of the chain stiffness $K$, for an irrational choice of $\left(r_{+}, r_{-}\right)$, and two values of $N$, defining a relatively short chain $(N=15)$ and one of intermediate length $(N=100)$. We find that $w$ is generally a complicated function of $K$, with flat plateaus and regimes of continuous evolution, not unlike what is found in the infinite-size limit studied through PBC simulations [1].

To investigate the peculiarities brought about by finite size, we analyze the dynamics for a large number of values of $\left(r_{+}, r_{-}\right)$and $K$, and observe that: (i) one or more velocity plateaus as a function of $K$ occur for large ranges of $\left(r_{+}, r_{-}\right)$; (ii) the velocity ratio $w$ of most plateaus satisfies

$$
w=1-\frac{1}{r_{+}^{\text {eff }}} .
$$

This result should be compared with the relation $\left(w=1-r_{+}^{-1}\right)$ valid for the main plateau of $\mathrm{PBC}$ calculations [1], where the length ratios $\left(r_{+}, r_{-}\right)$ are fixed: in Eq. (3) the new effective length ratio $r_{+}^{\text {eff }}$ of Eq. (2) replaces $r_{+}$. Figure 3 collects the observed plateau velocity ratios for a range of values of $r_{+}$and for fixed $r_{-}=\sqrt{101}$. Clear trends emerge: plateau velocities depend continuously on $r_{+}$, thus indicating that also the effective chain length ratio $r_{+}^{\text {eff }}$ evolves continuously with $r_{+}$in finite ranges. Several plateaus appear to follow different curves.

These data are conveniently organized and understood by plotting $r_{+}^{\text {eff }}$ rather than $w$, as a function of $r_{+}$, as is done in Fig. $4 .{ }^{1}$ All points fit perfect straight lines through $\left(r_{+}, r_{+}^{\text {eff }}\right)=(0,1)$. Most lines have slope $q / r_{-}$with integer $q$. Occasional plateaus fit this relation with half-integer $q(-5 / 2,7 / 2$, and $9 / 2$, in the calculations of Fig. 4).

Several calculations carried out with different values of $r_{-}$confirm that in general the ratio $r_{+}^{\text {eff }}$ satisfies

$$
r_{+}^{\mathrm{eff}}=1+q \frac{r_{+}}{r_{-}}
$$

with $q$ taking simple fraction (often integer) values. This general behavior indicates that the plateau dynamics leads the finite-size lubricant toward a dynamical configuration where not only its velocity but also its length is quantized.

1 For reasons of numerical stability, this figure reports $r_{+}^{\text {eff }}$ obtained by inversion of Eq. (3), but the same plot could have been obtained directly by applying the definition (2). This equivalence is confirmed by the comparison of the two methods shown in Fig. 4 for 2 points. 
We can understand this phenomenology in terms of kinks (i.e., local compressions of the chain with substrate potential minima holding more than just one particle [11]), as described in [1]. Assume initially integer $q$. The basic hypothesis explaining the relation (4) are: (i) the particles tend to singly occupy the $a_{+}$-spaced minima of the bottom potential, with occasional kinks to release the spring tension; (ii) kinks group in bunches, each sitting in a period $a_{-}$of the top substrate; (iii) kink bunches are $q$-fold, i.e. they collect $q$ individual kinks (negative $q$ indicates the number of anti-kinks). After the initial transient the chain length becomes on average very close to $L=\left(N-N_{k i n k}-1\right) a_{+}$. The number of kinks thus equals the number $q$ of kinks per bunch times the total number $L / a_{-}$of bunches in the chain: $N_{k i n k}=q L / a_{-}$. By eliminating $N_{k i n k}$, we obtain

$$
L=\frac{a_{+} a_{-}(N-1)}{a_{-}+q a_{+}} .
$$

This is consistent with an average inter-particle distance

$$
a_{0}^{\mathrm{eff}}=\frac{L}{N-1}=\frac{a_{+} a_{-}}{a_{-}+q a_{+}}=a_{+} \frac{r_{-}}{r_{-}+q r_{+}}=a_{+} \frac{1}{1+q \frac{r_{+}}{r_{-}}},
$$

and thus with the effective length ratio of Eq. (4). In general, for non-integer $q=n_{k} / n_{-}$values, this interpretation remains valid: bunches of a total of $n_{k}$ kinks distribute themselves in $n_{-}$minima of the $a_{-}$lattice. $q$ indicates therefore the density (coverage fraction) of kinks on the $a_{-}$lattice.

The dynamically stable plateau attractors of the open-boundary chain are therefore characterized by a lattice of kinks perfectly commensurate to the $a_{-}$ lattice. In the infinite-size PBC model, a rational kink coverage corresponds to commensurate encounter frequencies $f_{+}$and $f_{-}$of the generic lubricant particle with the two substrates, which in turn occurs for very special values of $r_{ \pm}$[1]: these $\left(r_{+}, r_{-}\right)$values are characterized by perfectly periodic singleparticle dynamics. It is rather remarkable that the open-chain model, without the necessity of any careful fine tuning of $\left(r_{+}, r_{-}\right)$realizes a self-organized commensurate kink lattice automatically producing perfectly periodic singleparticle oscillations in a generally incommensurate context.

Note that the points of Fig. 4 lying along the $q=0$ line indicate perfect commensuration between the lubricant chain and the bottom substrate, to which the chain remains pinned: this can be realized by paying a moderate harmonic-energy cost only close to $r_{+}=1$. For the same reason, all plateaus appear close to the $r_{+}=r_{+}^{\text {eff }}$ line (dot-dashed line in Fig. 4).

For the parameters of Fig. 3, nontrivial plateaus are found only for $0.7<$ $r_{+}<1.6$. Outside this range, relation (4) produces $r_{+}^{\text {eff }}$, for small integer $q$, 
very much different from $r_{+}$. Smaller values of $r_{-} \simeq 2$ (rather $r_{-} \simeq 10$ as in Fig. 3) generates an analogous set of plateaus for $r_{+}>1.6$.

\section{Size dependence}

As Fig. 2 suggests, strong size effects are observed, especially for large $K$. In particular, the "natural" symmetric large- $K$ limit $w=1 / 2$ found with PBC in Ref. [1] is rarely reached using OBC: for $N=100$ the chain is pinned to the bottom substrate $\left(v_{\mathrm{cm}}=0\right)$, while for $N=15$ it moves at $v_{\mathrm{cm}}=v_{\text {ext }}$. Figure 5 shows the dependence of the velocity ratio $w$ on the particle number $N$, for a fixed length ratio $r_{+}$and two different values of $r_{-}$, in the large- $K$ limit. We observe that the chain is pinned to the $a_{+}$substrate when $N$ occurs to be (nearly) a multiple of the length ratio $r_{-}$. In all other cases, the chain follows the $a_{-}$substrate, at velocity $v_{\text {ext }}$.

This changing behavior can be understood as follows. For large spring stiffness $K$, the kink dynamics is suppressed, the lubricant particles placing themselves at nearly regular distances $\simeq a_{0}$. It is energetically favorable for a chain shorter than $a_{-}$to sit in a minimum of the top potential and then stick to it. Even if the chain is longer than $a_{-}$, its length is generally not an exact multiple of the periodicity of the top substrate. For this reason, a finite end part of the chain remains similarly trapped in the minima of the top substrate: this is what occurs for most $N$ values in Fig. 5 . On the other hand, when $N a_{0}$ is a multiple of the $a_{-}$period (i.e. when $N$ is close to a multiple of $r_{-}=a_{-} / a_{0}$ ), minima and maxima of the upper potential compensate each other, so that there is no preferential relative position of chain and top substrate. For such special sizes, the chain remains weakly pinned to the bottom substrate, as illustrated in Fig. 5. The values $N=15$ and $N=100$ of Fig. 2 represent the two situations.

Other intermediate values occur for specific sizes, but the finite-size scaling for large $N$ is obviously non-trivial. While Fig. 2 indicates that for small and moderate $K$, size effects, if any, are very small, at large $K$ they affect the dynamics substantially. Further work is needed to understand the large- $K$ size-scaling in detail.

\section{Discussion and Conclusions}

We have shown that chains of finite and even small size, driven in between two periodic sliders, move with characteristic quantized velocities, much like the infinite-size ones do. We find that a finite-size chain length re-adjusts in such 
a way as to realize a lattice of topological solitons (kinks) commensurate to the period of the smoother slider. A consequence of this self-commensuration is a periodic single-particle motion, even for incommensurate initial choices of the periods.

The likely reason behind this phenomenology is as follows. Consider initially a single periodic slider of length ratio $r_{+}$and periodic boundary conditions. The lubricant chain will then form a regular lattice of kinks, which repel each other. When the second slider is introduced, the lattice of kinks will be generally incommensurate with the period $a_{-}$, and this brings an irregular distribution of bunches of kinks, which on average reconstruct the correct density of kinks. An OBC finite chain is able to relax, by paying some extra harmonic strain, in such a way as to enforce an optimal local density of kinks which satisfies both periodic sliders.

The phenomena just described for a model 1D system are unique, and it would be interesting if they could be observed in real systems. Nested carbon nanotubes [12], or confined one-dimensional nanomechanical systems [13], are one possible arena for the phenomena described. Though speculative at this stage, one obvious question is what aspects of the phenomenology just described might survive in two-dimensions (2D), where tribological realizations, such as the sliding of two hard crystalline faces with, e.g., an interposed graphite flake, are conceivable. Our results suggests that the lattice of discommensurations - a Moiré pattern- formed by the flake on a substrate, could be dragged by the other sliding crystal face, in such a manner that the speed of the flake as a whole would be smaller, and quantized. This would amount to the slider "ironing" the kinks onward. Dienwiebel et al. [3] demonstrated how incommensurability may lead to virtually friction-free sliding in such a case, but no measure was obtained for the flake relative sliding velocity. Real substrates are, unlike our model, not rigid, subject to thermal expansion, etc. Nevertheless the ubiquity of plateaus shown in Fig. 2, and their topological origin, suggests that these effects would not remove the phenomenon.

\section{Acknowledgments}

This research was partially supported by PRRIITT (Regione Emilia Romagna), Net-Lab "Surfaces \& Coatings for Advanced Mechanics and Nanomechanics" (SUP\&RMAN) and by MIUR Cofin 2004023199, FIRB RBAU017S8R, and RBAU01LX5H. 


\section{References}

[1] A. Vanossi, N. Manini, G. Divitini, G. E. Santoro, and E. Tosatti, Phys. Rev. Lett. 97, 056101 (2006).

[2] G. E. Santoro, A. Vanossi, N. Manini, G. Divitini, and E. Tosatti, Surf. Sci. 600, 2726 (2006).

[3] M. Dienwiebel, G. S. Verhoeven, N. Pradeep, J. W. M. Frenken, J. A. Heimberg, and H. W. Zandbergen, Phys. Rev. Lett. 92, 126101 (2004).

[4] M. G. Rozman, M. Urbakh, and J. Klafter, Phys. Rev. Lett. 77, 683 (1996); Europhys. Lett. 39, 183 (1997).

[5] M. G. Rozman, M. M. Urbakh, J. Klafter, and F.-J. Elmer, J. Phys. Chem. B 102, 7924 (1998).

[6] V. Zaloj, M. Urbakh, and J. Klafter, Phys. Rev. Lett. 81, 1227 (1998).

[7] A. E. Filippov, J. Klafter, and M. Urbakh, Phys. Rev. Lett. 87, 275506 (2001).

[8] O. M. Braun, A. Vanossi, and E. Tosatti, Phys. Rev. Lett. 95, 026102 (2005).

[9] T. S. van Erp, A. Fasolino, O. Radulescu, and T. Janssen, Phys. Rev. B 60, $6522(1999)$.

[10] A. Vanossi, J. Röder, A. R. Bishop, and V. Bortolani, Phys. Rev. E 63, 017203 (2000).

[11] O. M. Braun and Yu. S. Kivshar, The Frenkel-Kontorova Model: Concepts, Methods, and Applications, Springer-Verlag, Berlin (2004).

[12] X. H. Zhang, U. Tartaglino, E. Tosatti (unpublished).

[13] B. Toudic, F. Aubert, C. Ecolivet, P. Bourges, and T. Breczewski, Phys. Rev. Lett. 96, 145503 (2006). 


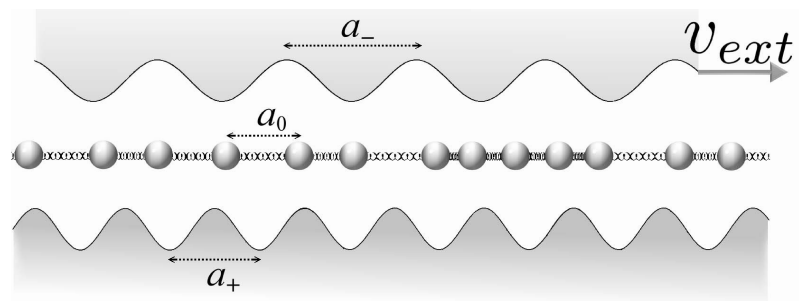

Fig. 1. (Color online) A sketch of the model with the two periodic sliders (periods $a_{+}$and $\left.a_{-}\right)$and the lubricant harmonic chain of rest nearest-neighbor length $a_{0}$.

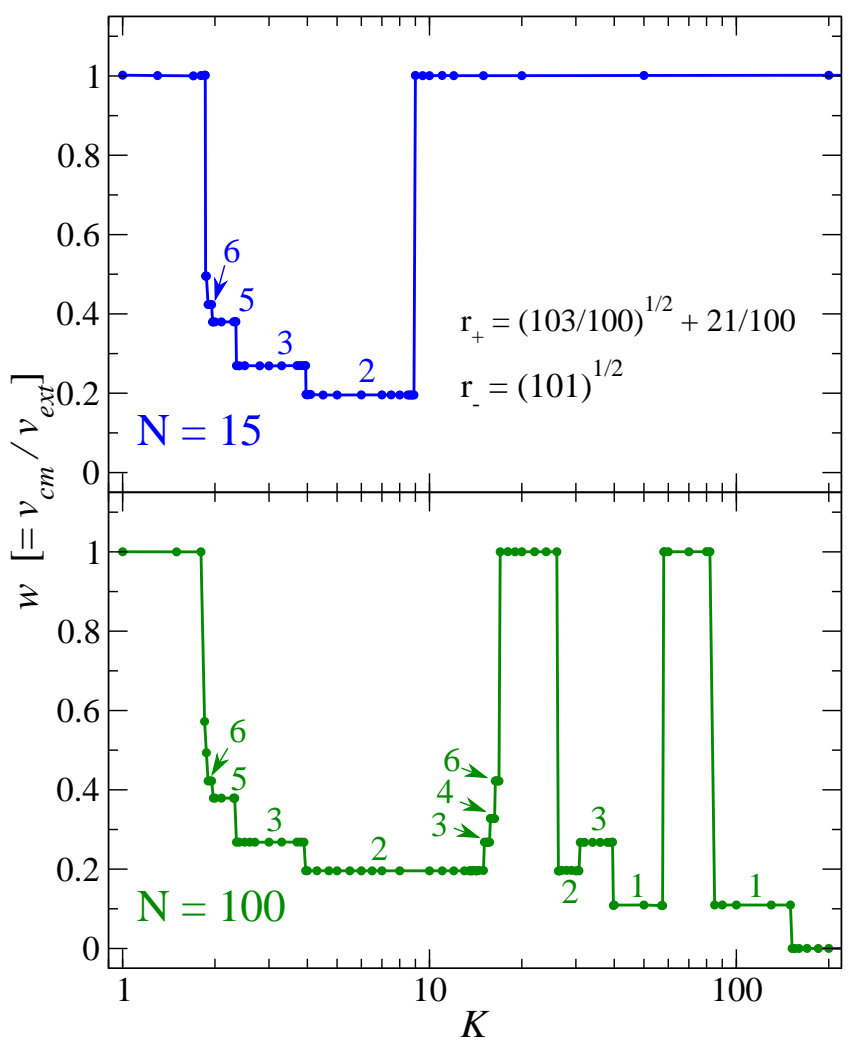

Fig. 2. (Color online) Average drift velocity ratio $w=v_{\mathrm{cm}} / v_{\text {ext }}$ as a function of the lubricant chain spring stiffness $K$ for $\left(r_{+}, r_{-}\right)=\left(\sqrt{\frac{103}{100}}+\frac{21}{100}, \sqrt{101}\right) \simeq(1.2249,10.050)$ and for two different finite sizes of the chain $N=15$ and 100 . OBC are applied, and $\gamma=0.1, v_{\text {ext }}=0.01$. The plateau labeling refers to values of $q$, defined in Eq. (4). Note the similar behaviour for $K<10$ in spite of the different chain size, and the different limit for large $K$, due to finite-size effects. 


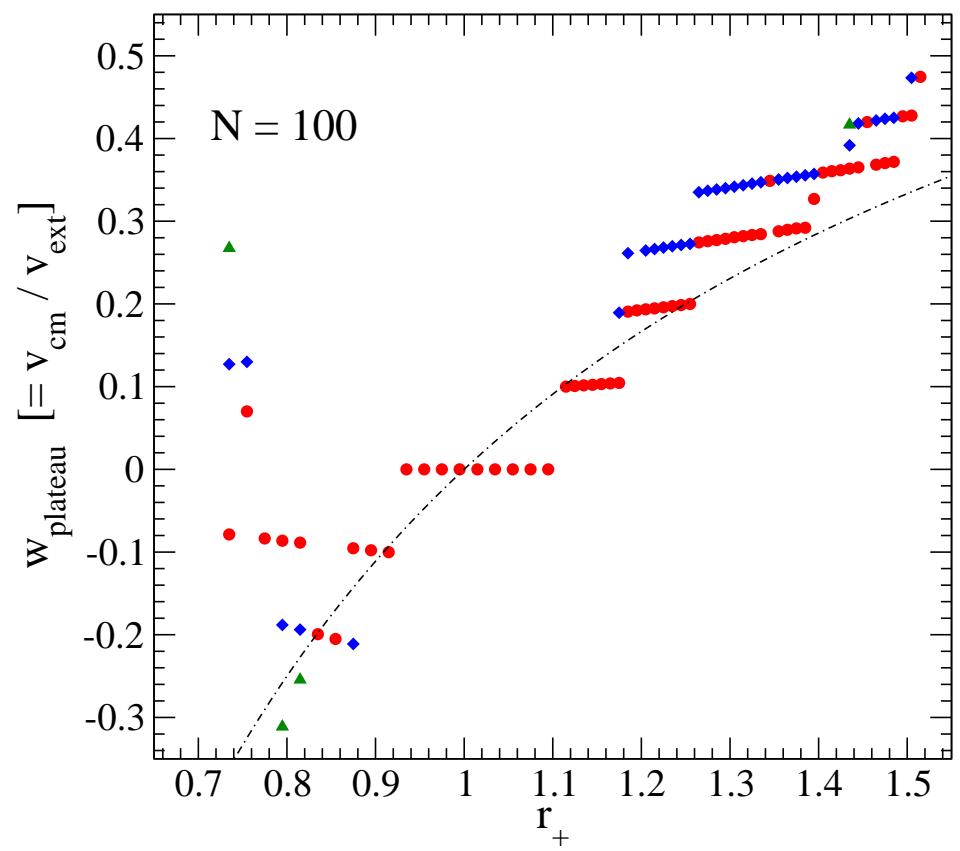

Fig. 3. (Color online) The plateau velocity ratio $w$ as a function of $r_{+}$, for $r_{-}=101^{1 / 2}$. Circles, diamonds and triangles represent respectively the first, second and third plateau found for increasing $|w|$. For comparison, we report the plateau velocity ratio $w=1-r_{+}^{-1}$ (dot-dashed curve) of the fixed-length $\mathrm{PBC}$ calculations [1]. Here $\gamma=0.1, v_{\text {ext }}=0.01$, and a finite chain of $N=100$ particles (OBC) is considered. 


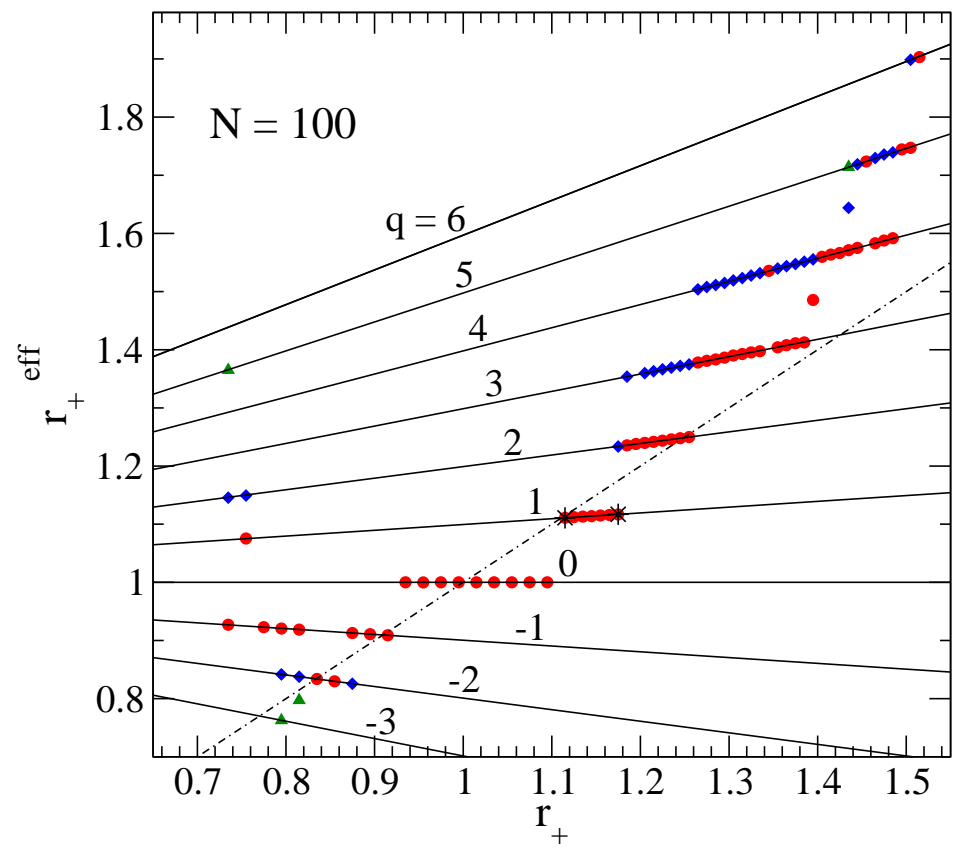

Fig. 4. (Color online) The effective length ratio $r_{+}^{\text {eff }}=a_{+} / a_{0}^{\text {eff }}$, calculated from the velocity data of Fig. 3 using $r_{+}^{\text {eff }}=(1-w)^{-1}$, the inverse of Eq. (3). The stars are $r_{+}^{\text {eff }}$ values obtained based on the definition (4) by time-averaging the chain length, showing perfect agreement with the velocity-derived values. The dot-dashed line represents the identity $r_{+}^{\text {eff }}=r_{+}$(undeformed chain length). Solid straight lines: $r_{+}^{\mathrm{eff}}=1+q \frac{r_{+}}{r_{-}}$, for integer $q$. 


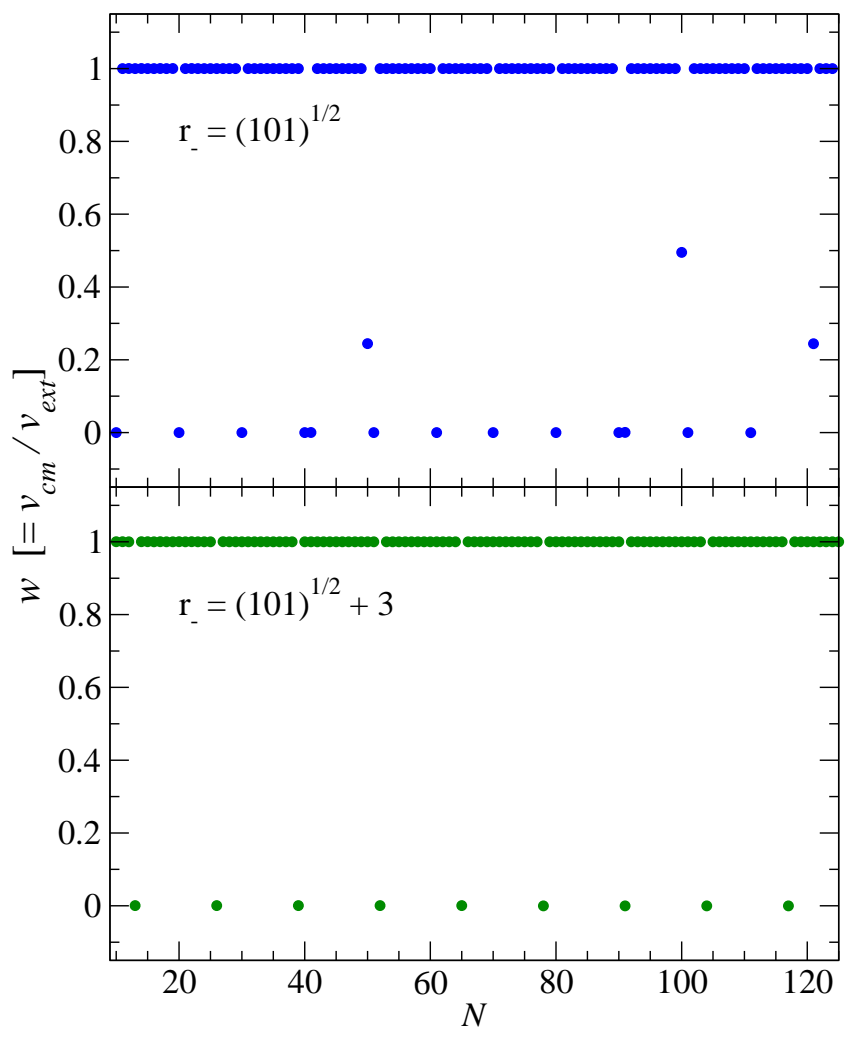

Fig. 5. (Color online) Size scaling of the average drift velocity ratio $w$ for $r_{+}=\frac{\sqrt{5}+1}{2}=\phi$, and two different values of the ratio $r_{-}=(101)^{1 / 2} \simeq 10.05$ and $r_{-}=(101)^{1 / 2}+3 \simeq 13.05$. The number of particles $N$ for which the chain is pinned to the $a_{+}$substrate $(w=0)$ are close to integer multiples of $r_{-}$. All calculations refer to $\gamma=0.1, v_{\text {ext }}=0.01$, and $K=1000$, a representative of the large- $K$ limit. 